\title{
DISKUSSION
}

\section{Diskussionsbeitrag zur Arbeit „Kompression des Plexus brachialis bei zwei Anomalien in der Skale- nuslücke" von G. Penkert}

(Acta chir. Austriaca 13,6 [1981])

\section{W. Sandmann}

Medizinische Einrichtungen der Liniversität Düsseldorf, Chirurgische Klinik und Poliklinik, Klinik A (Direktor: Prof. Dr. K. Kremer), Moorenstraße 5, D-4000 Düsseldorf

$\mathrm{Zu}$ diesem Fallbericht ist zu bemerken:

1. Der geschwungene Verlauf der A subclavia dextra ist eine Variante und keine Anomalie in der Skalenuslücke.

2. Die effektivste Therapic zur Behandlung des Plexusbrachialis-Kompressionssyndroms besteht in der Resektion der 1. Rippe mit Durchtrennung des M. scalenus anterior. Eine gleichzeitig vorhandene Halsrippe sollte auf jeden Fall reseziert werden. Dic Resektion der 1. Rippe ist notwendig, weil an dieser fibröse Stränge von der Halsrippe, vom dorsalen Anteil der 1. Rippe sowie vom M. scalenus anterior ansetzen können, welche das Kompressionssyndrom verursachen.

D. B. Roos beschreibt in diesem Zusammenhang 5 verschiedenen Typen des „thoracic outlet syndrome“. Werden nur diese Bänder sowie der $M$. scalenus anterior durchtrennt, so besteht durch erneute Bildung fibröser Stränge zur 1. Rippe eine erhebliche Rezidivrate.

3. Aus gefäßchirurgischer Sicht sind die Überlegungen des Autors, man habe wegen der Verlaufsvariante der $A$. subclavia die Halsrippe nicht resezieren können, ebenso unverständlich wie die fehlende Erwägung der Resektion der 1. Rippe. Dies hätte zu einer vollständigen Dekompression des Brachialisplexus geführt und eine mikrochirurgische Behandlung des $\mathrm{C}_{7} / \mathrm{C}_{8}$-Plexus unnötig gemacht. In diesem Zusammenhang muß noch bemerkt werden, da $B$ das Literaturverzeichnis eher antike Arbeiten zur Behandlung des ,thoracic outlet syndrome" betrachtet und wichtige moderne Beiträge außer acht läßt und darïber hinaus ziemlich unvollständig ist.
4. Der mikrochirurgische Gedanke als Therapiebeitrag zur Behandlung des Brachialiskompressionssyndroms hat sicherlich interessante Aspekte, sollte jedoch erst zum Tragen kommen, wenn ein nachgewiesenermaßen effektives und erprobtes Verfahren, wie die Resektion der 1. Rippe, Banddurchtrennung und Skalenotomie, nicht zum Therapieerfolg geführt hat. Inwieweit mikrochirurgische Maßnahmen dann noch helfen können, ist unseres Erachtens noch völlig offen. Jedenfalls geht der Wert des mikrochirurgischen Verfahrens aus dem Beitrag nicht hervor. Durch den Aspekt der Mikrochirurgie in diesem Beitrag kommt es eher zu einer technischen Fokussierung mit Gesichtsfeldeinschränkung bei Außerachtlassen makroskopisch lösbarer Probleme. Ist dies eine Blüte der zunehmend technisierten Medizin?

\section{Literatur}

(1) Crawford, F. A.: The thoracic outlet syndrome Surgical Clinics North America 60, 947 (1980).

(2) Gruss, J. D., Bartels, D., Kawai, S, Karadedos, C., Tsafanakis, E Straubel, H., Ohta, T.: Das Thoracic outlet Syndrom. angió 2, 77 (1980).

(3) Roos, D. B.: Thoracic outlet and carpal tunnel syndromes. In: Vascular Surgery. Ed. R B. Rutherford, p. 605. Saunders, Philadelphia 1977.

(4) Roos, D. B.: Transaxillary First Rib Resection for Thoracic Outlet Syndrome. In: Operative techniques in vascular surgery. Eds. J. J. Bergan, J. T. Yao, p. 125. Grune \& Stratton, New York 1980.

(5) Urschel, H. C., Razzuk, M. A. Albers, J. E: Reoperation for recurrent thoracic outlet syndrome Ann. Thoracic Surg. 21, 19 (1976). 